\title{
Anorectal Melanoma
}

\author{
David Row, M.D. ${ }^{1}$ and Martin R. Weiser, M.D. ${ }^{1}$
}

\section{ABSTRACT}

The authors review the epidemiologic, clinicopathologic, and molecular features of anorectal melanoma, and discuss the differences between this uncommon and lethal disease and the more common and curable cutaneous form. Observations regarding outcomes after surgical resection will also be discussed, as well as predictors of survival and the use of adjuvant therapies. The recent discovery that the KIT oncogene may be aberrantly activated in a subset of patients with anorectal melanoma creates a realm of possibility for the development of targeted molecular therapy. Melanoma of the anorectum is an extremely rare tumor that is often difficult to diagnose. Its surgical management is controversial, and the prognosis remains poor.

KEYWORDS: Melanoma, anorectal disease, molecular therapy, adjuvant treatment

Objectives: On completion of this article the reader should be able to summarize the management of anorectal melanoma.

Anorectal melanoma is a rare tumor with an extremely poor prognosis. It represents less than $1 \%$ of all melanomas and accounts for $\sim 4 \%$ of anal malignancies. ${ }^{1}$ The clinical diagnosis of anal melanoma is, at best, challenging. Patients often present with nonspecific complaints such as rectal bleeding (most common) or anal pain. Furthermore, a significant minority ( 34\%) of lesions may lack pigment, thus requiring biopsy for a definitive diagnosis. Although many patients present with what initially appears to be curable localized disease, undetected regional or distant metastases are common. Even with aggressive surgical resection, more than $80 \%$ of patients will die of distant metastatic disease within 5 years.

\section{EPIDEMIOLOGY}

The American Cancer Society estimates that $\sim 62,480$ new melanomas will be diagnosed in the United States in 2008 and 8,420 individuals will die of disease. Although the U.S. incidence rates of melanoma increased sharply in the 1970s and showed additional slow increases in the 1980s and 1990s, they have remained relatively stable since 2000 .

All melanomas originate from the melanocyte, a pigmented, dendritic-like cell found in a wide array of anatomic sites including the epidermis, eye, oropharyngeal epithelium, nasal cavity, vagina, urinary tract, and anus. Cutaneous melanomas are by far the most common form of the disease, comprising more than $90 \%$ of all melanomas. Although cutaneous melanomas account for a small minority (3\%) of skin malignancies, they cause a vast majority of skin cancer-related deaths. Of the remaining $(<10 \%)$ forms of melanoma, ocular melanoma accounts for $\sim 5 \%$, melanoma of unknown origin for $2 \%$, and mucosal melanoma for $1 \%$. Within the mucosal subgroup, head and neck, anorectal, female genital, and urinary tract tumors account for 55\%, 24\%, 18\%, and $3 \%$, respectively. ${ }^{2}$

\footnotetext{
${ }^{1}$ Colorectal Service, Department of Surgery, Memorial Sloan-Kettering Cancer Center, New York, New York.

Address for correspondence and reprint requests: Martin R. Weiser, M.D., Colorectal Service, Department of Surgery, Memorial Sloan-Kettering Cancer Center, 1275 York Ave., Rm. C-1075, New York, NY 10021 (e-mail: weiser1@mskcc.org).
}

Anal Cancer and Retrorectal Tumors; Guest Editor, Jan Rakinic, M.D. Clin Colon Rectal Surg 2009;22:120-126. Copyright (C) 2009 by Thieme Medical Publishers, Inc., 333 Seventh Avenue, New York, NY 10001, USA. Tel: +1(212) 584-4662.

DOI 10.1055/s-0029-1223844. ISSN 1531-0043. 


\section{Patient Characteristics}

Despite a shared cellular origin, there are distinct epidemiological differences between the cutaneous and noncutaneous forms of melanoma. Mucosal melanomas are commonly diagnosed in older individuals, with $\sim 50 \%$ of patients presenting in the $8^{\text {th }}$ decade of life or older; in contrast, only $\sim 25 \%$ of patients with cutaneous melanoma present when older than 70. Race, ethnicity, and gender differences also exist. In the United States, fewer than $3 \%$ of cutaneous melanomas are diagnosed within the African American and Hispanic populations; however, these minority groups represent $9 \%$ of individuals diagnosed with mucosal melanomas. Women are more likely than men to be diagnosed with anorectal melanoma (male:female ratio $0.75,95 \%$ confidence interval $[\mathrm{CI}]=0.57$ to 0.90$){ }^{3}$ Nevertheless, the fact that women are more likely to undergo perineal examination as part of a routine health maintenance checkup confounds this apparent female predilection and raises the question of actual differences in tumor biology.

Sixty-five percent of anorectal melanomas are located within the anal canal or at the anal verge; however, disease is also identified in the distal rectum in $35 \%$ of cases. ${ }^{4}$ Given this, it is not surprising that the presenting symptoms often consist of bleeding, anorectal discomfort, an appreciable anorectal mass, or change in bowel habits (Fig. 1). Diagnosis is often delayed because, as noted previously, identifiable lesions are pigmented in only about one-third of cases. In addition, many believe that the disease itself is characterized by an aggressive biology; at the time of correct diagnosis, a majority of patients have advanced lesions measuring $>2 \mathrm{~mm}$ in thickness. ${ }^{5}$ On occasion, incidental melanomas are identified during pathologic examination of a hemorrhoidectomy or anal polyp specimen.

Noncutaneous melanomas are more likely than cutaneous melanomas to be diagnosed at an advanced

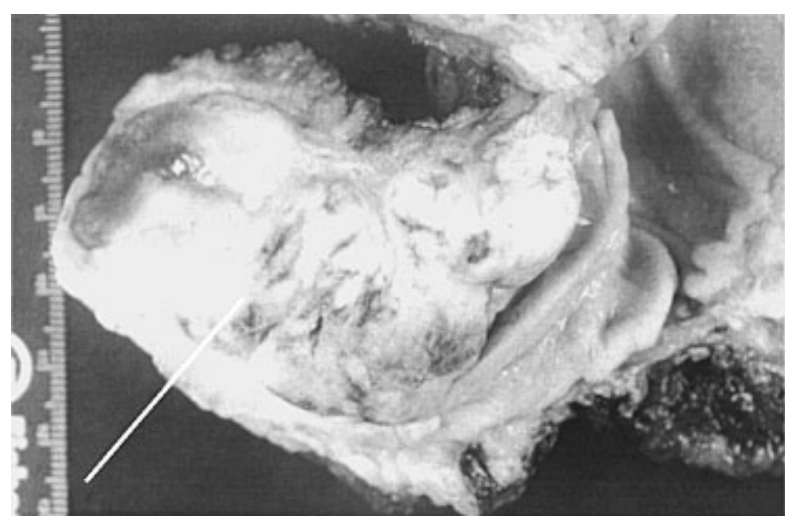

Figure 1 Bisected abdominoperineal resection specimen showing a large nonpigmented melanoma of the anal canal (white arrow). Photograph courtesy of Dr. Jinru Shia, Department of Pathology, Memorial Sloan-Kettering Cancer Center. stage. The incidence rates for locoregional lymph node metastases on initial presentation are $61 \%$ for anorectal melanomas, $21 \%$ for head and neck melanomas, $23 \%$ for melanomas of the female genital tract, and $11 \%$ for urinary tract melanomas. This is in contrast to the $9 \%$ of cutaneous melanomas with lymph node metastases on initial presentation. At the time of diagnosis, distant metastases are identified in as many as $29 \%$ of patients. ${ }^{2}$

\section{Risk Factors}

In the United States, race is a strong predictor for development of melanoma. Although the rates of cutaneous and ocular melanomas are 5 to 20 times higher in Caucasians than in African Americans, rates of mucosal melanomas are only twice as high. These findings suggest that cutaneous and ocular melanomas may share a common environmental risk factor (i.e., ultraviolet radiation) that does not promote the development of mucosal melanomas. Sun exposure is not a risk factor for mucosal melanoma, and individuals with darker skin pigmentation have a higher incidence of anorectal melanoma.

Interestingly, there is indirect evidence that infection with the human immunodeficiency virus (HIV) may increase the risk for development of anorectal melanoma. ${ }^{4}$ This raises the question of an association between anorectal melanoma and immunosuppression, which will be addressed in the subsection on immunotherapy.

\section{MOLECULAR PATHOGENESIS}

\section{KIT Receptor Tyrosine Kinase}

The KIT receptor tyrosine kinase plays an integral role in the normal development and function of melanocytes during embryonic and postnatal stages of growth. Lossof-function mutations in KIT and its ligand result in white spotting phenotypes in mice and humans. Activating $K I T$ mutations have also been implicated in the pathogenesis of leukemia and gastrointestinal stromal tumors (GISTs). More recently, KIT aberrations have been associated with the pathogenesis of malignant melanoma.

KIT expression is typically detected in normal melanocytes (benign nevi and in situ melanomas), but it appears to be downregulated in the more invasive and metastatic forms of melanoma. A progressive decrease in KIT immunopositivity is associated with an increase in dermal invasiveness. It has been hypothesized that KIT signaling impacts the regulation of cell differentiation and tissue morphogenesis; thus, tumor progression may necessitate loss of KIT expression. In addition, reports of activating KIT mutations (and resultant protein overexpression) seen in a subset of melanoma patients point 
toward a different mechanism through which the constitutive activation of $K I T$, via oncogenic mutations, promotes mitogenesis and subsequent neoplastic transformation.

The genetic alterations associated with melanomas of different sites, along with varying degrees of sun exposure, imply the presence of distinct molecular pathways in their development. This may significantly impact potential molecular treatment of the disease. The indepth molecular pathogenesis of KIT and other gene mutations (i.e., $B R A F, N R A S)$ implicated in malignant melanoma is beyond the scope of this review. A recent study by Antonescu et $\mathrm{al}^{6}$ demonstrated that anorectal melanomas show an increased prevalence of activating KIT mutation. Additional studies focusing on the development of potential therapeutic molecular targets are warranted.

\section{TREATMENT OF ANORECTAL MELANOMA}

Despite important advances made in delineating the molecular pathogenesis of anorectal melanoma, and the potential for utilizing this knowledge in the research and development of new therapies, surgery remains the mainstay of treatment. The role of adjuvant chemotherapy and immunotherapy in the treatment of anorectal melanoma is yet to be established. The optimal extent of surgery (wide local excision versus radical resection) and extent of lymphadenectomy remain controversial.

\section{Extent of Surgical Resection}

There is debate in the medical literature regarding the extent of surgery required for optimal treatment of primary anorectal melanoma. Early studies suggested that aggressive treatment (abdominoperineal resection) of the primary lesion was associated with improved outcome, possibly because of the regional lymphadenectomy that is inevitably part of this radical resection. ${ }^{7}$ However, other studies have reported similar patterns of recurrence and survival, and no significant increase in local failure, in patients undergoing local excision of the primary without regional lymphadenectomy. ${ }^{8,9}$ A common conclusion reached by all studies is that relapses are usually distant and lethal.

Because of the rarity of this disease, treatment guidelines are based on small retrospective studies. The benefits of local excision are obvious, and include quicker recovery time from a significantly less invasive procedure, minimal impact on bowel function, and avoidance of a permanent colostomy. Nonetheless, local excision does not address the issue of potential metastatic involvement of the regional lymph node basin, which is one of the most important predictors of outcome in primary cutaneous melanoma.
This issue was previously addressed in a study of 56 patients with localized anorectal melanoma who were treated at Memorial Sloan-Kettering Cancer Center by either abdominoperineal resection or local excision between 1929 and 1993. The study indicated a possible survival advantage in patients who had undergone regional lymph node resection, as 9 of 10 long-term survivors were in the radical surgery group. ${ }^{7}$ Of note, two long-term survivors-one in the radical resection group and one in the local excision group (who subsequently underwent therapeutic pelvic lymphadenectomy)-had positive mesenteric lymph nodes on pathologic examination. Interestingly, the rate of isolated local recurrence was comparable in both groups. Additional studies examining local excision of anorectal melanoma have not reported high rates of isolated regional relapse, supporting the hypothesis that local recurrence is not the cause of patient death. ${ }^{10}$

In a more recent series from Memorial SloanKettering Cancer Center, Yeh et $\mathrm{a}^{11}$ reported a change in practice patterns within that institution over time. In their study of 46 patients with anorectal melanoma over a 20 -year period, the authors noted a paradigm shift in treatment strategy, with local excision trumping radical resection (Table 1). They reported that, between 1984 and 1996, 15 of 21 (71\%) patients treated for primary anorectal melanoma had abdominoperineal resection. From 1997 to 2003, however, 21 of 25 (84\%) patients treated for anorectal melanoma underwent local excision. During these respective periods, no changes were seen in either patient demographics or in the thickness and diameter of the tumors. Patients treated with radical resection tended to present with thicker lesions as compared to those treated with local excision: median tumor thickness was $11 \mathrm{~mm}$ (range, 1.1 to $26 \mathrm{~mm}$ ) in the abdominoperineal resection group versus $7.2 \mathrm{~mm}$ (range, 1.1 to $19 \mathrm{~mm}$ ) in the local excision group; however, this difference was not statistically significant. Despite the clear change in practice patterns during these two periods, the outcomes were identical (Fig. 2): 75\% of patients recurred.

Yeh et $\mathrm{al}^{11}$ analyzed outcome data based on extent of surgery. Five-year disease-specific survival for the entire cohort was 34\%, with a median follow-up of 39 months for survivors. Thirty-four of 46 patients relapsed within 10 months, with an overall recurrence rate of $53 \%$ at 1 year. As noted previously, the majority of patients developed distant recurrences. No differences were seen in patterns of relapse between patients treated by radical resection versus local excision: 5 of 19 (26\%) patients in the abdominoperineal resection group and 7 of $27(26 \%)$ in the local excision group developed local recurrence as the first sign of relapse. Survival was similar in both groups, with a 5-year disease-specific survival of $32 \%$ for the abdominoperineal resection cohort and 35\% for the local excision cohort (Fig. 3). These findings 
Table 1 Primary Tumor, Treatment, and Outcome in Patients Treated Before and After 1997

\begin{tabular}{|c|c|c|c|c|}
\hline & Total $(n=46)$ & $\begin{array}{l}1984-1996 \\
(n=21)\end{array}$ & $\begin{array}{l}1997-2003 \\
(n=25)\end{array}$ & $P$ \\
\hline Surgery & & & & $<0.0001$ \\
\hline APR & $19(41 \%)$ & $15(71 \%)$ & $4(16 \%)$ & \\
\hline LE & $27(59 \%)$ & $6(29 \%)$ & $21(84 \%)$ & \\
\hline Thickness & & & NS & \\
\hline$\leq 10 \mathrm{~mm}$ & $30(70 \%)$ & $11(58 \%)$ & $19(79 \%)$ & \\
\hline$>10 \mathrm{~mm}$ & $13(30 \%)$ & $8(42 \%)$ & $5(21 \%)$ & \\
\hline \multicolumn{5}{|c|}{ First site of relapse } \\
\hline All sites & $34(74 \%)$ & $16(76 \%)$ & $18(72 \%)$ & NS \\
\hline Locoregional & $12(26 \%)$ & $7(33 \%)$ & $5(20 \%)$ & \\
\hline Distant & $13(28 \%)$ & $6(29 \%)$ & $7(28 \%)$ & \\
\hline Both & $9(20 \%)$ & $3(14 \%)$ & $6(24 \%)$ & NS \\
\hline
\end{tabular}

APR, abdominoperineal resection; LE, local excision; NS, not significant.

From Yeh et al. ${ }^{11}$ Used with permission of Lippincott Williams \& Wilkins.

suggest that local recurrence and survival in anorectal melanoma are not associated with extent of resection.

\section{Inguinal Lymphadenectomy}

The need for regional lymphadenectomy in the surgical treatment of anorectal melanoma is also a subject of debate. Mesorectal, pelvic sidewall, and inguinal lymph nodes are at increased risk for metastases from anorectal lesions. In an abdominoperineal resection, the mesorectal lymph nodes are resected en bloc with the primary tumor. However, Yeh et $\mathrm{al}^{11}$ found that lymph node metastases did not predict outcome in patients undergoing radical resection. Based on this finding, nodal disease in the setting of anorectal melanoma may not have the same biologic significance as nodal disease in the setting of cutaneous melanoma.

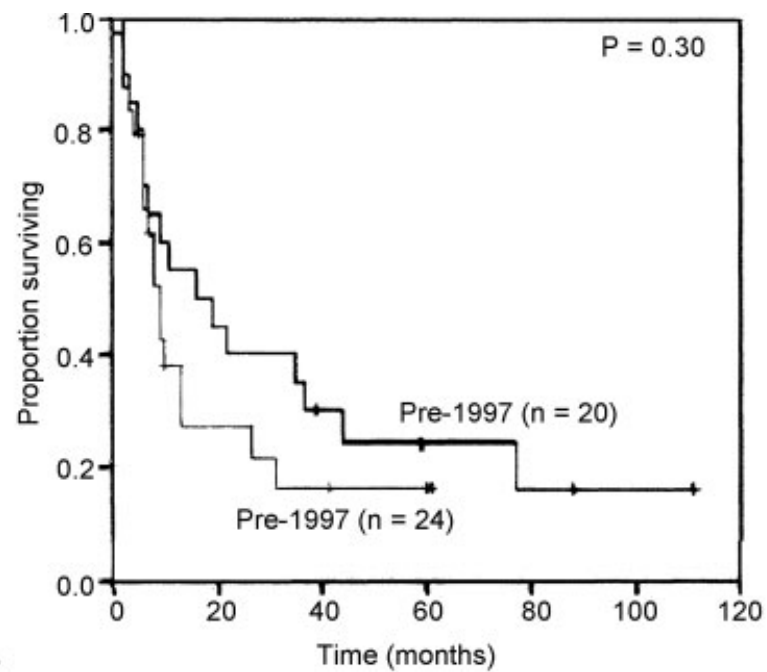

Prophylactic bilateral inguinal lymphadenectomy in patients with clinically nonpalpable lymph nodes has not been shown to improve survival, and carries an increased risk of complications. For clinically palpable disease, elective inguinal lymph node dissection should be considered. Sentinel lymph node analysis in anorectal melanoma has been studied. However, due to the uncertainty surrounding the prognostic significance of nodal disease, its role in anorectal melanoma has yet to be defined.

Unfortunately, the prognosis for anorectal melanoma is poor regardless of treatment modality. Many experts have hypothesized that this is the result of early systemic dissemination that takes place in tumorigenesis, and that micrometastases are well established at the time of initial clinical detection and diagnosis. Although there are currently no clear data

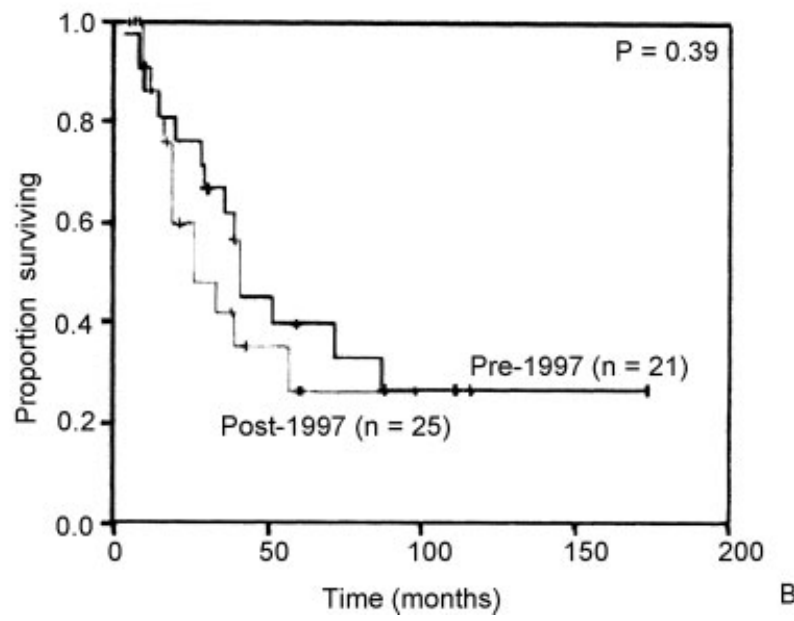

Figure 2 Changes in practice at Memorial Sloan-Kettering Cancer Center (MSKCC): (A) Recurrence-free survival before and after 1997. (B) Disease-specific survival before and after 1997. From Yeh et al. ${ }^{11}$ Used with permission of Lippincott Williams \& Wilkins 


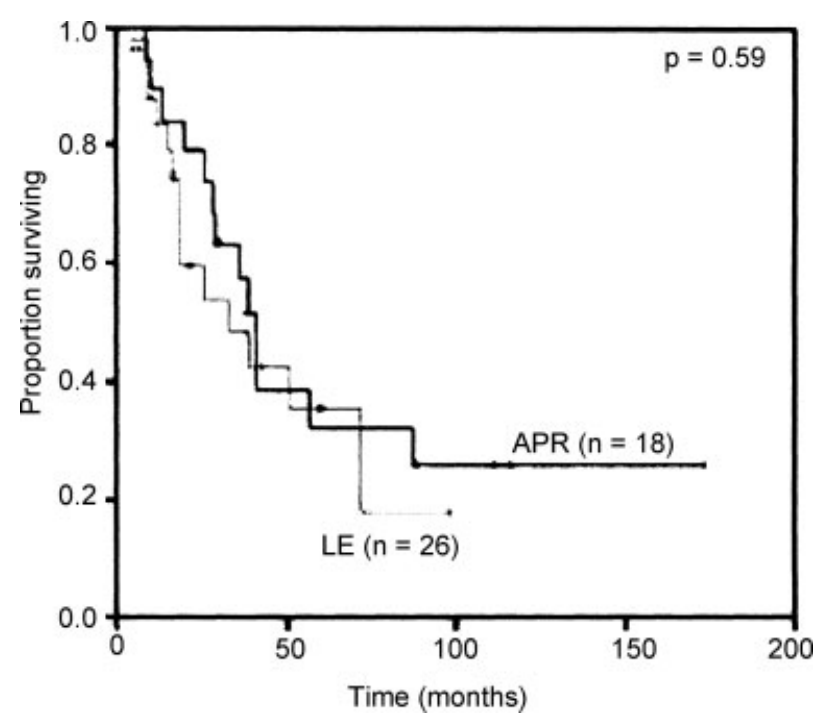

Figure 3 Disease-specific survival in patients who underwent abdominoperineal resection (APR) compared with local excision (LE). From Yeh et al. ${ }^{11}$ Used with permission of Lippincott Williams \& Wilkins.

supporting the use of adjuvant therapies for anorectal melanoma, continued investigation and documented case reports provide hope that successful adjuvant agents will be identified in the future. ${ }^{12}$

\section{Adjuvant Therapy}

\section{CHEMOTHERAPY}

Cytotoxic chemotherapy is generally not an effective treatment for patients with cutaneous melanoma. Experiential use of chemotherapy in the setting of advanced anorectal melanoma has been limited to subsets of patients in larger retrospective reviews, and it has often been given in combination with immunotherapeutic agents and radiation therapy. Dacarbazine, the most widely used single agent, is associated with a response rate of $\sim 20 \%$. The vast majority of responses, however, are partial, with median response duration of only 4 to 6 months. There are no prospective, randomized, controlled trial data supporting a survival benefit for dacarbazine versus best supportive care or placebo. ${ }^{13}$ Temozolomide, an analog of dacarbazine with similar activity, has the benefit of being available orally, and may be associated with lower rates of central nervous system relapse.

Dacarbazine or temozolomide in combination with a variety of other agents has demonstrated no advantage over single-agent chemotherapy in phase III trials. In one large multicenter trial, 240 patients were randomized to either dacarbazine or a four-drug combination known as the Dartmouth regimen (dacarbazine, cisplatin, carmustine, and tamoxifen). The Dartmouth regimen exhibited a marginally improved response rate (19\%) over dacarbazine alone (10\%), but this was not found to be statistically significant. Both treatment arms had a median survival of only 7 months. Toxicities such as bone marrow suppression, nausea/vomiting, and fatigue were more severe for patients receiving the combination therapy. ${ }^{14}$

\section{IMMUNOTHERAPY}

Melanoma shows a greater susceptibility to immune assault than most other tumor types, and has therefore been the most widely studied target for immunotherapy. As seen on histopathologic examination, melanomas are typically infiltrated with lymphocytes; the absence of tumor-infiltrating lymphocytes is associated with a poorer prognosis. The absence of a primary tumor in $\sim 5 \%$ of patients with metastatic melanoma hints at an immune-mediated regression of the primary lesion. A minority of patients with metastatic disease demonstrates a response to immune mediators such as interferon- $\alpha$ and interleukin-2, suggesting that metastatic melanoma is also susceptible to immune assault. Additionally, primary melanoma is more common in immunosuppressed patients, who have an overall increased risk of developing other cancers.

Tumor cells from melanomas can be grown in vitro with relative ease, permitting the identification of tumor antigens and the development of immunotherapeutic modalities such as monoclonal antibodies and antigen-directed T-cells. These developments have further resulted in the evolution and utilization of a large number of different targeted treatment strategies for melanoma. These include vaccines that induce humoral immunity and enhance cellular immunity, inflammatory cytokines, and immune modulators such as thalidomide.

\section{RADIATION THERAPY}

Melanoma has historically been considered a relatively radioresistant tumor, although more recent data challenges this viewpoint. In vitro studies of melanoma cell lines have demonstrated widely varying sensitivities to radiation, ${ }^{15}$ raising the possibility that some melanoma cells exhibit a type of radioresponsiveness similar to that shown by "late-reacting" or slow-renewal normal tissues. Such tumors require a greater-than-standard dose per radiation fraction for greatest therapeutic effect. ${ }^{16}$ Radiation is now more commonly included as a component in the treatment armamentarium.

In a recent study from M.D. Anderson Cancer Center, 23 patients with anorectal melanoma underwent local excision (including lymph node dissection of documented regional nodal disease) followed by adjuvant radiation therapy. ${ }^{17}$ The radiation was delivered using a hypo-fractionated regimen of $30 \mathrm{~Gy}(5$ fractions over 2.5 weeks). Nine patients received adjuvant systemic therapy. The actuarial 5-year local control rate 
was $74 \%$, with an actuarial 5-year disease-specific survival of only $36 \%$. Undoubtedly, radiation is an effective palliative therapy for the 40 to $50 \%$ of patients who develop unresectable, locally recurrent, and/or metastatic disease resulting in bone pain, epidural spinal cord compression, tumor hemorrhage, and central nervous system dysfunction secondary to cerebral involvement.

\section{PROGNOSIS}

Five-year survival rates according to anatomic location are $80 \%$ for cutaneous melanoma, $75 \%$ for ocular melanoma, and $20 \%$ for anorectal mucosal melanoma. There are several possible reasons for the generally poorer prognosis of anorectal melanoma: delay in diagnosis, inherent increased aggressiveness of tumor biology, and earlier dissemination of disease as a result of close contact with the rich lymphovascular supply of the underlying anorectal mucosa.

In their study of 46 patients undergoing surgical resection for anorectal melanoma, Yeh et $\mathrm{al}^{11}$ reported a 5 -year disease-specific survival of $34 \%$ with a median follow-up of 39 months. Thirty-four of the 46 patients who relapsed did so within a median 10-month period, with a recurrence rate of $53 \%$ at 1 year. The majority of patients developed distant recurrent disease.

The strongest predictor of outcome in this series was the histopathologically confirmed presence of perineural invasion in the primary tumor (Fig. 4). Of the 8

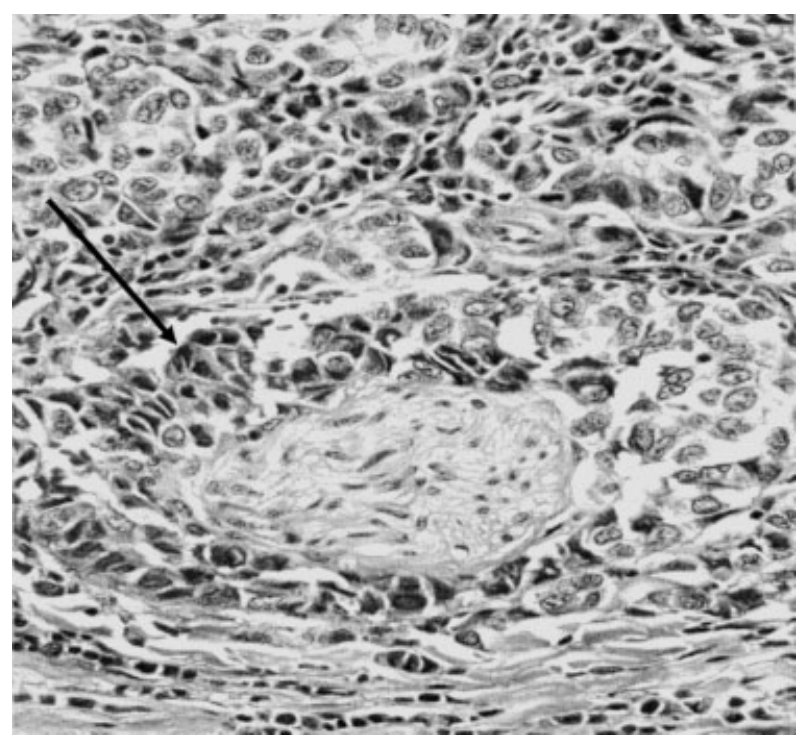

Figure 4 Hematoxylin and eosin (H\&E) stained histological section of an anorectal melanoma showing epithelioid tumor cells arranged in a nested growth pattern. The black arrow points to the presence of perineural invasion where tumor cells grow tightly around a peripheral nerve branch. Photograph courtesy of Dr. Jinru Shia, Department of Pathology, Memorial Sloan-Kettering Cancer Center.

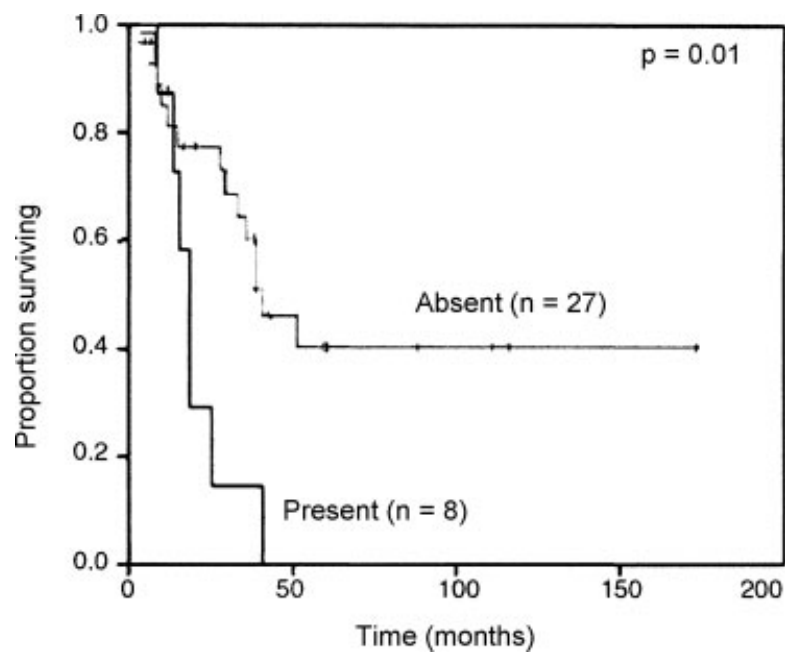

Figure 5 Disease-specific survival in patients with tumor perineural invasion. From Yeh et al. ${ }^{11}$ Used with permission of Lippincott Williams \& Wilkins.

patients with perineural invasion $100 \%$ recurred, compared with $67 \%$ of the 29 patients without perineural invasion. For the cohort with perineural invasion, median survival was 19 months versus 39 months for the cohort without perineural invasion (Fig. 5). At last follow-up, only 1 of 8 patients (13\%) with perineural invasion was alive with disease, but this individual had lung, liver, and perirectal lymph node metastases; whereas 10 of 29 patients (34\%) without perineural invasion were alive with no evidence of disease. On multivariate analysis using Cox regression, perineural invasion was the only independent predictor of disease-specific survival (hazard ratio $3.4 ; 95 \%$ CI 1.2 to 9.9; $P=0.02$; Table 2). Factors such as regional nodal metastasis, tumor size, and tumor thickness were not associated with recurrence or survival in this series.

Because anorectal melanoma is an uncommon tumor, staging schemes have been limited to delineation of local, regional, and distant disease. The presence of perineural invasion by tumor, however, is an independent prognosticator that merits further exploration in future clinical studies.

Table 2 Multivariate Analysis of Prognostic Factors Associated with Survival

\begin{tabular}{lll}
\hline Variable & Hazard Ratio & $\boldsymbol{P}$ \\
\hline Perineural invasion & $3.4[1.2,9.9]$ & 0.01 \\
Symptoms & & 0.62 \\
Thickness & & 0.08 \\
Diameter & & 0.23 \\
Mural involvement & 0.33 \\
Necrosis & & 0.96 \\
\hline
\end{tabular}

From Yeh et al. ${ }^{11}$ Used with permission of Lippincott Williams \& Wilkins. 


\section{CONCLUSION}

Anorectal melanoma is a rare disease with a poor prognosis, even when apparently diagnosed at an early clinical stage and showing no evidence of distant disease. Anorectal mucosal melanoma differs on a molecular basis from cutaneous melanoma. With the recent discovery of KIT overexpression in a subset of patients, the possibility of developing new molecularly based therapeutic strategies now exists. Currently, however, surgery remains the mainstay of treatment. Local excision is recommended whenever technically feasible, with inguinal lymphadenectomy reserved for clinically palpable disease. The ultimate goal of surgical treatment should be to minimize patient morbidity and maximize quality of life. The role of adjuvant therapy in the treatment of anorectal melanoma continues to be defined.

\section{REFERENCES}

1. Klas JV, Rothenberger DA, Wong WD, Madoff RD. Malignant tumors of the anal canal: the spectrum of disease, treatment, and outcomes. Cancer 1999;85(8):16861693

2. Chong AE, Karnell LH, Menck HR. The National Cancer Data Base report on cutaneous and noncutaneous melanoma: a summary of 84,836 cases from the past decade. The American College of Surgeons Commission on Cancer and the American Cancer Society. Cancer 1998;83(8):16641678

3. McLaughlin CC, Wu XC, Jemal A, Martin HJ, Roche LM, Chen VW. Incidence of noncutaneous melanomas in the U.S. Cancer 2005;103(5):1000-1007

4. Cagir B, Whiteford MH, Topham A, Rakinic J, Fry RD. Changing epidemiology of anorectal melanoma. Dis Colon Rectum 1999;42(9):1203-1208
5. Wanebo HJ, Woodruff JM, Farr GH, Quan SH. Anorectal melanoma. Cancer 1981;47(7):1891-1900

6. Antonescu CR, Busam KJ, Francone TD, et al. L576P KIT mutation in anal melanomas correlates with KIT protein expression and is sensitive to specific kinase inhibition. Int J Cancer 2007;121(2):257-264

7. Brady MS, Kavolius JP, Quan SH. Anorectal melanoma. A 64-year experience at Memorial Sloan-Kettering Cancer Center. Dis Colon Rectum 1995;38(2):146-151

8. Ward MW, Romano G, Nicholls RJ. The surgical treatment of anorectal malignant melanoma. Br J Surg 1986;73(1):68-69

9. Thibault C, Sagar P, Nivatvongs S, Ilstrup DM, Wolff BG. Anorectal melanoma-an incurable disease? Dis Colon Rectum 1997;40(6):661-668

10. Bullard KM, Tuttle TM, Rothenberger DA, et al. Surgical therapy for anorectal melanoma. J Am Coll Surg 2003;196(2): 206-211

11. Yeh JJ, Shia J, Hwu WJ, et al. The role of abdominoperineal resection as surgical therapy for anorectal melanoma. Ann Surg 2006;244(6):1012-1017

12. Yeh JJ, Weiser MR, Shia J, Hwu WJ. Response of stage IV anal mucosal melanoma to chemotherapy. Lancet Oncol 2005;6(6):438-439

13. Crosby T, Fish R, Coles B, Mason MD. Systemic treatments for metastatic cutaneous melanoma. Cochrane Database Syst Rev 2000;(2):CD001215

14. Chapman PB, Einhorn LH, Meyers ML, et al. Phase III multicenter randomized trial of the Dartmouth regimen versus dacarbazine in patients with metastatic melanoma J Clin Oncol 1999;17(9):2745-2751

15. Rofstad EK. Radiation biology of malignant melanoma. Acta Radiol Oncol 1986;25(1):1-10

16. Bentzen SM, Overgaard J, Thames HD, et al. Clinical radiobiology of malignant melanoma. Radiother Oncol 1989; 16(3):169-182

17. Ballo MT, Gershenwald JE, Zagars GK, et al. Sphinctersparing local excision and adjuvant radiation for anal-rectal melanoma. J Clin Oncol 2002;20(23):4555-4558 